\title{
Active Treatment is Not Indicated in the Majority of Patients Diagnosed with a Vestibular Schwannoma: A Review on the Natural History of Hearing and Tumor Growth
}

\author{
Martin Nue Møller • Søren Hansen • \\ Hidemi Miyazaki $\cdot$ Sven-Eric Stangerup • \\ Per Caye-Thomasen
}

Published online: 12 September 2014

(C) Springer Science+Business Media New York 2014

\begin{abstract}
This article describes various epidemiologic trends for vestibular schwannomas over the last 40 years and provides updated information based on recent reviews and original work on the natural history of tumor growth and hearing level following the diagnosis of a vestibular schwannoma. A treatment strategy based on the natural history of tumor growth and hearing is proposed and discussed, with attention to the latest surgical and non-surgical developments in the field.
\end{abstract}

Keywords Acoustic neurinoma - Observation strategy · Spontaneous course $\cdot$ Wait and scan

\author{
M. N. Møller · S. Hansen · H. Miyazaki · S.-E. Stangerup · \\ P. Caye-Thomasen ( $\square)$ \\ Department of ORL, HNS and Audiology F2074, Copenhagen \\ University Hospital Rigshospitalet, Blegdamsvej 9, \\ 2100 Copenhagen, Denmark \\ e-mail: per.caye-thomasen.01@ regionh.dk \\ M. N. Møller \\ e-mail: martin@nuemoller.dk \\ S. Hansen \\ e-mail: sorenhansen1@gmail.com \\ H. Miyazaki \\ e-mail: miyazaki@jikei.ac.jp \\ S.-E. Stangerup \\ e-mail: stangerup@pc.dk \\ H. Miyazaki \\ Department of Otorhinolaryngology, The Jikei University, \\ 3-25-8 Nishi-shinbashi Minato-ku, Tokyo, Japan \\ P. Caye-Thomasen \\ Faculty of Medical and Health Sciences, University \\ of Copenhagen, Copenhagen, Denmark
}

\section{Introduction}

The incidence of vestibular schwannomas (VS, also called acoustic neurinoma) has increased from less than 3 per million/yr in the seventies to more than 20 per million/yr, as reported in recent studies based on the largest prospective and unbiased VS database worldwide, now exceeding 3,000 Danish patients [1,2]. The incidence increase is supported by findings in other countries [3,4] and the true incidence may prove to be even higher [2-4]. Arguably, the increase is at least in part due to improved diagnostic methods.

A large VS may be life threatening and surgery is thus equivocally advocated. However, several treatment modalities may be chosen is case of a small or medium-sized tumor, i.e., observation, surgery, or radiotherapy. Success of treatment is usually perceived to be control of tumor growth and preservation of a serviceable hearing level in patients presenting with good hearing, with no or minimal treatment side-effects. In order to justify invasive treatment either by surgery or radiotherapy for these smaller tumors the outcome should be superior to that resulting from the natural history of the disease. Only a detailed knowledge of the natural history of these tumors will allow a comparison with and a proper

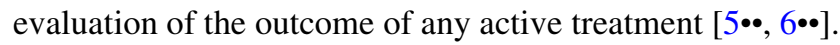

Accordingly, this review presents a comprehensive overview of the recent literature on the natural history of tumor growth pattern, as well as the spontaneous hearing outcome in observed unilateral vestibular schwannomas. Data on recent epidemiological trends are also presented and finally, a treatment strategy based on the natural history is discussed.

\section{Recent Epidemiological Trends}

The incidence of diagnosed VS has increased continuously throughout the recent decades, at least in part due to 
increased and easier access to improved diagnostic methods (MRI), as well as a reduction in the referral time for investigation of patients with unexplained unilateral audiovestibular symptoms reduced patient and doctors delay. A continued increase of patients diagnosed with VS is to be expected for the years to come, as the true incidence of the tumor is undoubtedly higher [2-4], as indicated by accidental tumor findings and temporal bone studies. Today, the tumors are found at an earlier stage, as demonstrated by the decrease of the diagnostic tumor size from a mean size of $35 \mathrm{~mm}$ in the late seventies to $10 \mathrm{~mm}$ in the period from 2003 to 2012 [1, 2, 7••, 8]. Accordingly, more and more purely intrameatal, as well as small and medium-sized extrameatal tumors are being diagnosed. As these are the patients with all three current management options, this trend emphasizes the need for, and relevance of, hard data on the natural history of the disease. The patient age at diagnosis has changed throughout the years as well, from a mean of 48 years in 1976 to 57 years in 2011 [1, 7••]. Thus, more and more tumors are being found in increasingly older patients, and the presenting tumor sizes are smaller and smaller.

\section{The Natural History of Vestibular Schwannoma Growth}

Although many original papers as well as reviews regarding the growth pattern of VS have been published, the natural history is still enigmatic. The overall conclusion is that the tumor may grow in by any conceivable pattern. Tumors may grow in a linear fashion, they may grow and then stop growing and can grow again, they may not grow and then grow, they may grow quickly and then only slowly or stop growing. A small percentage of tumours will regress over time. A tumor may show continued or periodic growth after diagnosis - and it may even display regress. Most commonly, though, no change of size is seen during many years of observation (by repeated MRI) after the diagnosis. A number of centers have explored clinical or other predictors of tumor growth, and although young age and relatively large tumor size at diagnosis may constitute growth predictors, no general agreement exists on this issue.

The reported percentages of vestibular schwannomas exhibiting growth range widely, from 6 to $73 \%[4,7 \bullet \bullet, 8-$ 12]. However, larger patient series, meta-analyses and reviews averages the occurrence of significant growth in 20-45\% after 3-5 years of observation (Fig. 1) [4, 7••, 9, $13,14]$. Annual growth rate ranges typically from 2 to $4 \mathrm{~mm}$, excluding the cases with significant regression or exceptional growth, the latter most often occurring in cystic tumors. Tumor regression or shrinkage is reported in $4-22 \%[4,7 \bullet \bullet, 8-14,15 \bullet]$.

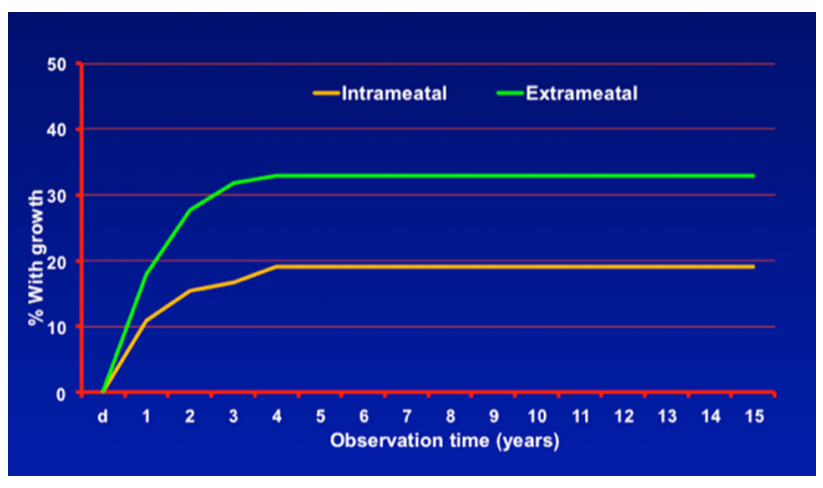

Fig. 1 Nelson-Aalen plot, depicting the cumulated risk of growth with observation in intrameatal and extrameatal vestibular schwannomas $(n=552)$. From ref. [8]

There is a general consensus in the literature that tumors are most likely to grow within the first few years after diagnosis. Thus, tumor growth occurring beyond the fifth year of observation is very rare, but is, however, still observed $\left[12,16^{\bullet}\right]$.

Most papers on tumor growth do not distinguish between purely intrameatal and tumors with an extrameatal extension. The few that do, however, report that the growth pattern of intrameatal tumors is different from the extrameatal ones.

\section{Growth of Intrameatal Tumors}

Very few recent studies have investigated the tumor growth pattern in a larger series of purely intrameatal VS. In the largest series of 156 patients, $45 \%$ displayed tumor growth during a mean of about 5 years of observation [12], which is seemingly more than the around $35 \%$ reported by most authors for extrameatal tumors with an equivalent followup period [7••]. However, only $19 \%$ of the intrameatal tumors grew to extrameatal extension (Fig. 1) [12], which from a clinical point of view may be considered the most crucial feature. Recent works from other centers show comparable results $[17,18 \bullet \cdot$. Sub-localization within or tumor-induced expansion of the meatus at diagnosis does not predict subsequent growth [12].

\section{Growth of Extrameatal Tumors}

The vast majority of published work on tumor growth is based on extrameatal tumors. Arguably, at least some of the extrameatal tumors may in fact have been intrameatal before diagnosis. Nevertheless, growth occurrence seems to be different in the extrameatal tumors (or when the tumor has become extrameatal), as a larger percentage exhibit growth, ranging between 29 and $54 \%$ [4, 7••, 9, 13, 14]. Thus, it seems that the risk of further growth is higher 
when the tumor has an extrameatal component at diagnosis. If growth occurs, this is likely to occur within the very first few years after diagnosis and this temporal pattern does not differ between purely intrameatal and extrameatal tumors (Fig. 1) $[4,7,8-14,15 \bullet 16 \bullet]$.

\section{Natural History of Hearing in Patients with Vestibular Schwannoma}

At diagnosis, around $90 \%$ of patients with a vestibular schwannoma have an asymmetrical sensorineural hearing loss, which makes unilateral hearing impairment the predominating symptom of VS. The hearing acuity is usually classified according to the AAO-HNS or the Gardner Robertson classification system, which includes the pure tone average (PTA) and speech discrimination (SD), or the word recognition score (WRS), which is based exclusive on SD. Most authors consider AAO-HNS class A and B, or Gardner Robertson grade I and II, or a WRS better than $50 \%$ as serviceable hearing, as a hearing aid may be applied with benefit for the patient. A SD better than $70 \%$ in combination with a PTA below $30 \mathrm{~dB}$ is considered to be good hearing (AAO-HNS class A, Gardner Robertson grade I).

Relating hearing to tumor size, the hearing quality at diagnosis has changed during the latter decades. In the late 1970s, $9 \%$ of patients had a PTA better than $30 \mathrm{~dB}$ at diagnosis and $21 \%$ had a $\mathrm{SD}>70 \%$, whereas this has changed over time to 23 and $57 \%$, respectively, in 2010 $[7 \bullet \bullet, 19]$. Accordingly, whereas the tumor size is decreasing, the hearing level at diagnosis is continuously

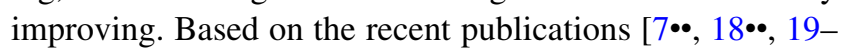
$21,22 \cdot, 23]$, half of the patients present with good hearing at diagnosis. After diagnosis, the hearing level will deteriorate faster in the tumor ear, compared with the contralateral ear, at a rate of approximately $3 \mathrm{~dB}$ PTA/year and $5 \%$ discrimination/year. The hearing will deteriorate faster in patients with growing, as opposed to stable or regressing tumors. In intrameatal tumors, subsequent hearing loss does not depend on sub-localization within the canal or tumor-induced canal expansion. On average, $60 \%$ of patients presenting with good hearing at diagnosis will maintain a good hearing level after 5 years of observation. Notably, patients with a normal SD at diagnosis will maintain a good hearing level which is markedly better than patients with only a small discrimination loss, and it is especially noteworthy that $75 \%$ maintains good hearing after 10 years (Fig. 2) [19, 20]. In other words, the rate of hearing loss is much lower for patients with normal discrimination at diagnosis. This is a significant clinical finding, as these patients are often recommended to have hearing preservation surgery, in order to preserve their

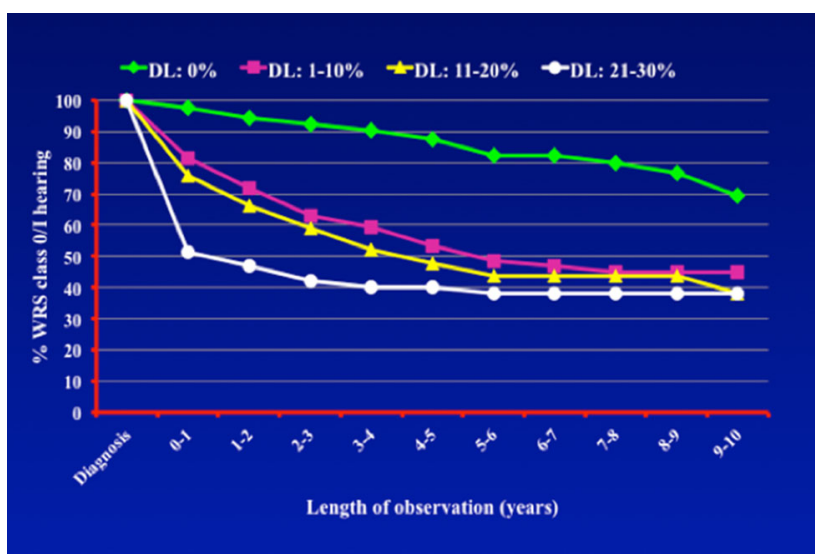

Fig. 2 Preservation of good hearing (WRS Class I) on the tumor ear in patients with a vestibular schwannoma during observation, in relation to the different SD subgroups at diagnosis $(n=491)$. From ref. [20]

good hearing. The reason for the good spontaneous hearing preservation in these patients remains elusive.

\section{Treatment Strategy}

Considering that only around one-third of small to medium-sized VS grows within 5 years after diagnosis and that very few tumors grow beyond this timeframe $[7 \bullet \bullet, 15 \bullet, 16 \bullet$, 17], the main argument in favor of primary active treatment (surgery or radiotherapy) is to preserve hearing function. However, reviews on published results of hearing preservation by both surgery and radiotherapy show that on average, around $50 \%$ of patients preserve good or serviceable hearing short-term by both modalities [24••, 25]. By observation, $60 \%$ preserve good hearing after 5 years and if the discrimination is normal at diagnosis, as many as $75 \%$ preserve good hearing after 10 years $[7,19,20]$. In other words, all patients with a small or medium-sized tumor should be offered watch and wait management primarily, by repeated MRI scans. If growth occurs, active treatment is indicated.

However, some specialized centers may have documented considerably better than average results of active treatment, thus justifying primary active treatment. The use of novel and improved intra-operative neuro-monitoring equipment (see below) may also improve functional results considerably, thus leading to primary surgery as a reasonable option for optimal hearing preservation. In addition, other reasons for microsurgery may exist, e.g., otherwise intractable dizziness or physiological reasons, although these are generally rare.

On a global scale, more and more centers are adopting the strategy of primary observation of small and mediumsized tumors. As growth after diagnosis occurs within the 
very first few years in almost all cases, the following observation strategy by repeated MRI to assess tumor size is suggested (after diagnosis): 6 months, 1, 2, 3, 4, 5 years, and then every other year until 10 years. After 10 years, termination may be considered, as growth after this duration is very rare, but we suggest scan every 5 years, until 25 years, in order to collect long-term data. Again, if significant growth occurs during observation, active treatment is recommended. Continued observation may be continued if the tumor is still small, or in patients with significant comorbidity. However, the patient should be informed, that continuing wait and scan management in the face of a growing tumor may increase the risk of morbidity from active treatment at a later date.

\section{Controversies}

Despite solid evidence that only around one-third of small to medium-sized vestibular schwannomas grow after diagnosis, the wait and scan management option may not be offered to patients universally and in some departments, therefore, there is a risk of the pursuance of active treatment with a concomitant risk of morbidity and its financial implications [5••]. In addition, a number of centers offering radiotherapy as primary treatment are taking the natural history of the disease into account for the success of their treatment $\left[60^{\bullet}\right]$, as they do not await documented growth before treatment. If $65-70 \%$ of the tumors do not grow as part of the natural course of the disease, then $90 \%$ tumor growth control by primary radiotherapy is a growth control improvement of only $20-25 \%$. In addition, $65-70 \%$ of the irradiated patients are over treated and subjected to the risks of radiation.

When reviewing the literature on the natural history of tumor growth and hearing in patients with vestibular schwannomas, as well as the literature on the tumor control and hearing outcome of both surgery and radiotherapy, it is evident that only very little long -term data exist. A follow-up of more than 3-5 years is only provided by very few centers and there is thus an emergent need for hard long-term outcome data, in order for the neuro-otologists, neuro-surgeons, and neuro-oncologists to be able to provide the patient with evidence based on the outcomes of different treatment modalities long-term. Thus, long-term clinical research on the subject is needed, for the patients to able to make an informed choice on the management of their vestibular schwannoma.

Existing data on the natural history and the various treatments available have primarily dealt with tumor control, facial nerve function, and hearing. Our knowledge on dizziness, vertigo, and unsteadiness in treated and untreated VS patients is comparably very scarce, and this is problematic, as compromised balance seems to be more detrimental for quality of life and vocational capabilities [26, 27•, 28•].
Future Perspectives

Gathering of long-term data on tumor growth and hearing by observation and outcome of the various treatment options, as well as data on quality of life and problems of balance is likely to change the perspective on the disease and its clinical management. In particular studies on the effect of impaired vestibular function are few, even though vestibular disturbance has a major impact on everyday activities and the ability to work.

Refined principles of radiotherapy and new developments in microsurgical techniques are likely to improve results and reduce morbidity. More specifically, emerging novel and improved systems for real-time intra-operative monitoring of the function of the facial and the cochlear nerve are very promising [29••], for improved functional preservation of these nerves. The systems may also aid in intra-operative decision-making, concerning the applicability of a cochlear implant in case of an anatomically preserved cochlear nerve with preserved electrical, but not acoustical function.

Medical treatment is now an option in the treatment of vestibular schwannomas associated with Neurofibromatosis type 2 (NF2), in the form of anti-VEGF. Tumor expression of VEGF (vascular endothelial growth factor) is correlated positively with tumor growth and anti-VEGF treatment has been shown to reduce tumor size and improve hearing in some patients with NF2 [30]. Although NF2-associated tumors usually are more aggressive, antiVEGF or other medical treatment is likely to present as an option for sporadic tumor treatment in the future.

\section{Conclusion}

The natural history of vestibular schwannomas remains the gold standard to which any active treatment outcome should be measured. Whereas surgical treatment of large tumors is undisputed, active treatment of small and medium-sized tumors should await documented growth, as only one-third of these tumors grow after diagnosis. However, long-term data on tumor control, hearing acuity and especially balance function are needed, for patients observed, operated or irradiated. Emerging novel and improved systems for real-time intra-operative monitoring of the function of the facial and the cochlear nerve are very promising, for improved functional preservation of these nerves. These systems may alter treatment strategies in the future, in favor of microsurgical tumor resection.

\section{Compliance with Ethics Guidelines}

Conflict of Interest $\quad$ Per Caye-Thomasen, Martin Nue Møller, Søren Hansen and Sven-Eric Stangerup declare that they have no conflict of 
interest. Hidemi Miyazaki reports non-financial support from Nihon Kohden, outside the submitted work.

Human and Animal Rights and Informed Consent This article does not contain any studies with human or animal subjects performed by any of the authors.

\section{References}

Papers of particular interest, published recently, have been highlighted as:

- Of importance

•• Of major importance

1. Stepanidis K, Kessel M, Caye-Thomasen P, Stangerup SE. Sociodemographic distribution of vestibular schwannomas in Denmark. Acta Otolaryngol. 2014;134(6):551-6.

2. Stangerup SE, Tos M, Thomsen J, Caye-Thomasen P. True incidence of vestibular schwannoma? Neurosurgery. 2010;67(5): 1335-40 discussion 1340.

3. Evans DG, Moran A, King A, Saeed S, Gurusinghe N, Ramsden R. Incidence of vestibular schwannoma and neurofibromatosis 2 in the North West of England over a 10-year period: higher incidence than previously thought. Otol Neurotol. 2005;26(1):93-7.

4. Bakkouri WE, Kania RE, Guichard JP, Lot G, Herman P, Huy PT. Conservative management of 386 cases of unilateral vestibular schwannoma: tumor growth and consequences for treatment. J Neurosurg. 2009;110(4):662-9.

5. • Miller T, Lau T, Vasan R, Danner C, Youssef AS, van Loveren $\mathrm{H}$, Agazzi S. Reporting success rates in the treatment of vestibular schwannomas: are we accounting for the natural history? J Clin Neurosci. $2014 ; 21(6): 914-8$. Draws appropriate attention to the problematic fact that a number of treatment centers partly accounts for the natural history of vestibular schwannoma growth when reporting results of radiotherapy.

6. • Lau T, Olivera R, Miller T Jr, Downes K, Danner C, van Loveren HR, Agazzi S. Paradoxical trends in the management of vestibular schwannoma in the United States. J Neurosurg. 2012;117(3): 514-9. Current trends in the treatment of vestibular schwannoma in the US were evaluated, by analysing data from a national cancer database, representing $26 \%$ of the population. Data from 2004 to 2007 were included, showing a decrease in resection and an increase in radiation in small to medium-sized tumors. The observation strategy showed a modest increase, but remained low at an average of $25 \%$, leading the authors to assert that vestibular schwannomas are being overtreated in the US.

7. • Stangerup SE, Caye-Thomasen P. Epidemiology and natural history of vestibular schwannomas. Otolaryngol Clin North Am. 2012;45(2):257-68, vii. Comprehensive and updated review of the epidemiology and natural history from the world's largest database on patients with vestibular schwannoma (in Copenhagen, Denmark).

8. Stangerup SE, Caye-Thomasen P, Tos M, Thomsen J. The natural history of vestibular schwannoma. Otol Neurotol. 2006;27(4):547-52.

9. Nikolopoulos TP, Fortnum H, O'Donoghue G, Baguley D. Acoustic neuroma growth: a systematic review of the evidence. Otol Neurotol. 2010;31(3):478-85.

10. Hughes M, Skilbeck C, Saeed S, Bradford R. Expectant management of vestibular schwannoma: a retrospective multivariate analysis of tumor growth and outcome. Skull Base. 2011;21(5): 295-302.

11. Moffat DA, Kasbekar A, Axon PR, Lloyd SK. Growth characteristics of vestibular schwannomas. Otol Neurotol. 2012;33(6):1053-8.
12. Caye-Thomasen P, Hansen S, Dethloff T, Stangerup SE, Thomsen J. Sublocalization and volumetric growth pattern of intracanalicular vestibular schwannomas. Laryngoscope. 2006;116(7): 1131-5.

13. Al Sanosi A, Fagan PA, Biggs ND. Conservative management of acoustic neuroma. Skull Base. 2006;16(2):95-100.

14. Yoshimoto Y. Systematic review of the natural history of vestibular schwannoma. J Neurosurg. 2005;103(1):59-63.

15. - Huang X, Caye-Thomasen P, Stangerup SE. Spontaneous tumour shrinkage in 1261 observed patients with sporadic vestibular schwannoma. J Laryngol Otol. 2013;127(8):739-43. Shows that in 1261 observed patients, tumor regression was seen in $4 \%$.

16. - Fayad JN, Semaan MT, Lin J, Berliner KI, Brackmann DE. Conservative management of vestibular schwannoma: expectations based on the length of the observation period. Otol Neurotol. 2014 Jun 9. [Epub ahead of print]. Of 114 conservatively managed patients, as many as $86 \%$ retained serviceable hearing after 4 years and only one-third had to undergo further treatment.

17. Lee JD, Park MK, Kim JS, Cho YS. The factors associated with tumor stability observed with conservative management of intracanalicular vestibular schwannoma. Otol Neurotol. 2014;35(5):918-21.

18. • Pennings RJ, Morris DP, Clarke L, Allen S, Walling S, Bance ML. Natural history of hearing deterioration in intracanalicular vestibular schwannoma. Neurosurgery. 2011;68(1):68-77. In 47 patients with purely intracanalicular tumors, $74 \%$ maintained good hearing after 3.6 years, according to the 50/50 rule. The authors conclude, that hearing results with conservative management appear similar to those reported for stereotactic radiotherapy or microsurgery.

19. Caye-Thomasen P, Dethloff T, Hansen S, Stangerup SE, Thomsen J. Hearing in patients with intracanalicular vestibular schwannomas. Audiol Neurootol. 2007;12(1):1-12.

20. Stangerup SE, Thomsen J, Tos M, Cayé-Thomasen P. Long-term hearing preservation in vestibular schwannoma. Otol Neurotol. 2010;31(2):271-5.

21. Stangerup SE, Tos M, Thomsen J, Caye-Thomasen P. Hearing outcomes of vestibular schwannoma patients managed with 'wait and scan': predictive value of hearing level at diagnosis. J Laryngol Otol. 2010;124(5):490-4.

22. - Sughrue ME, Kane AJ, Kaur R, Barry JJ, Rutkowski MJ, Pitts LH, Cheung SW, Parsa AT. A prospective study of hearing preservation in untreated vestibular schwannomas. J Neurosurg. 2011 Feb;114(2):381-5. This paper on 59 patients confirms previous reports on larger cohorts, showing that tumor growth is associated with a faster rate of hearing loss.

23. Sughrue ME, Yang I, Aranda D, Lobo K, Pitts LH, Cheung SW, Parsa AT. The natural history of untreated sporadic vestibular schwannomas: a comprehensive review of hearing outcomes. J Neurosurg. 2010;112(1):163-7.

24. •• Yang I, Sughrue ME, Han SJ, Aranda D, Pitts LH, Cheung SW, Parsa AT. A comprehensive analysis of hearing preservation after radiosurgery for vestibular schwannoma: clinical article. J Neurosurg. 2013;119 Suppl:851-9. This systematic literature review shows that an overall hearing preservation rate of $\sim 51 \%$ is to be expected 3-4 years after radiotherapy.

25. Sughrue ME, Yang I, Aranda D, Kane AJ, Parsa AT. Hearing preservation rates after microsurgical resection of vestibular schwannoma. J Clin Neurosci. 2010;17(9):1126-9.

26. Lloyd SK, Kasbekar AV, Baguley DM, Moffat DA. Audiovestibular factors influencing quality of life in patients with conservatively managed sporadic vestibular schwannoma. Otol Neurotol. 2010;31(6):968-76.

27. - Breivik CN, Varughese JK, Wentzel-Larsen T, Vassbotn F, Lund-Johansen M. Conservative management of vestibular 
schwannoma-a prospective cohort study: treatment, symptoms, and quality of life. Neurosurgery. 2012;70(5):1072-80; discussion 1080. 192 patients were followed prospectively and their quality of life assessed by two validated questionnaires, showing that vertigo was found to significantly reduce quality of life.

28. - Breivik CN, Nilsen RM, Myrseth E, Finnkirk MK, Lund-Johansen M. Working disability in Norwegian patients with vestibular schwannoma: vertigo predicts future dependence. World Neurosurg. 2013;80(6):e301-5. The prospective series of 206 Norwegian patients found that the generally overlooked vestibular complaints were significant predictors for becoming dependant of disability pension.

29. •• Oh T, Nagasawa DT, Fong BM, Trang A, Gopen Q, Parsa AT, Yang I. Intraoperative neuromonitoring techniques in the surgical management of acoustic neuromas. Neurosurg Focus. 2012;33(3):E6. The authors reviews and analyses the major intra-operative neuro-monitoring techniques available for vestibular schwannoma resection, demonstrating a great degree of recent success in this regard. Although this is a recent paper, it was not possible to include information on a brand new system called the CPA Master, which introduces mapping and real-time monitoring of both the cochlear and facial nerve, using specially designed electrodes to be placed directly on the brain stem.

30. Plotkin SR, Merker VL, Halpin C, Jennings D, McKenna MJ, Harris GJ, Barker FG 2nd. Bevacizumab for progressive vestibular schwannoma in neurofibromatosis type 2: a retrospective review of 31 patients. Otol Neurotol. 2012;33(6):1046-52. 